\title{
Prevalência de ansiedade em profissionais da saúde em tempos de COVID-19: revisão sistemática com metanálise
}

\author{
Prevalence of anxiety among health professionals \\ in times of COVID-19: a systematic review with meta-analysis
}

David Franciole Oliveira Silva (https://orcid.org/0000-0003-0940-1356) ${ }^{1}$

Ricardo Ney Cobucci (https://orcid.org/0000-0002-0184-2061) ${ }^{2}$

Vanessa de Paula Soares-Rachetti (https://orcid.org/0000-0002-9551-8771) ${ }^{3}$

Severina Carla Vieira Cunha Lima (https://orcid.org/0000-0001-8268-1986) ${ }^{4}$

Fábia Barbosa de Andrade (https://orcid.org/0000-0002-7055-8726) ${ }^{1}$
${ }^{1}$ Programa de Pós-Graduação em Saúde Coletiva, Universidade Federal do Rio Grande do Norte (UFRN). Av. Sen. Salgado Filho 1787, Lagoa Nova. 59056-000 Natal RN Brasil.davfranci@ hotmail.com

${ }^{2}$ Programa de PósGraduação em

Biotecnologia, Curso de Medicina, Universidade Potiguar (UnP). Natal RN Brasil.

${ }^{3}$ Departamento de Biofísica e Farmacologia, Centro de Biociências, UFRN. Natal RN Brasil.

${ }^{4}$ Programa de Pós-

Graduação em Nutrição,

UFRN. Natal RN Brasil.

\begin{abstract}
This study sets out to identify the prevalence of anxiety among health professionals during the COVID-19 pandemic. It involves a systematic review and meta-analysis of studies published in any language in 2020. A search was conducted in the Embase, LILACS and PubMed databases using the keywords anxiety, COVID-19, health workers, and synonyms. The estimated overall prevalence of anxiety with a 95\% confidence interval was calculated using the random effects model. Of the 861 records identified, 36 articles were included in the systematic review and 35 in the meta-analysis. The overall prevalence of anxiety was 35\% (95\%CI: 29-40). A higher risk of anxiety was identified among women compared to men (Odds Ratio: 1.64 [95\%CI: 1.47-1.84]), and in nurses, in comparison with physicians (Odds Ratio: 1.19 [95\%CI: 1.07-1.33]). Being on the front line of COVID-19, being infected with coronavirus and having chronic diseases were also factors associated with a higher risk of anxiety. A high prevalence of anxiety among health professionals was observed, with higher risk among women and nurses. There is a pressing need for measures aimed at prevention of anxiety and providing early and appropriate treatment for those suffering from moderate and severe anxiety.

Key words Anxiety, Pandemic, COVID-19, Health professionals, Systematic review
\end{abstract}

Resumo O objetivo deste estudo é identificar a prevalência de ansiedade em profissionais de saúde durante a pandemia da COVID-19. Trata-se de revisão sistemática de estudos publicados em qualquer idioma em 2020. Foi realizada busca nas bases de dados Embase, LILACS e PubMed utilizando os descritores anxiety, COVID-19, health workers, e sinônimos. A estimativa da prevalência geral de ansiedade com intervalo de confiança de 95\% foi calculada utilizando o modelo de efeitos aleatórios. Dos 861 registros identificados, 36 artigos foram incluídos na revisão sistemática e 35 na metanálise. A prevalência geral de ansiedade foi de 35\% (IC95\%: 29-40). Foi identificado maior risco de ansiedade nas mulheres em relação aos homens (Odds Ratio: 1.64 [IC95\%: 1,47-1,84]), e nos enfermeiros, na comparação com médicos (Odds Ratio: 1.19 [IC95\%: 1,07-1,33]). Atuar na linha de frente no combate a COVID-19, estar infectado com coronavirus e apresentar doenças crônicas também foram fatores associados com maior risco de ansiedade. Observa-se alta prevalência de ansiedade entre profissionais de saúde, com maior risco entre mulheres e enfermeiros. Há necessidade de medidas que visem sua prevenção, bem como o fornecimento de tratamento precoce e adequado aos com ansiedade moderada e grave. Palavras-chave Ansiedade, Pandemia, COVID-19, Profissionais de saúde, Revisão sistemática 


\section{Introdução}

A pandemia da COVID-19 (doença causada pelo novo coronavírus) é atualmente o mais grave problema de saúde pública a ser enfrentado no mundo ${ }^{1}$. Segundo dados da Organização Mundial da Saúde ${ }^{2}$, até meados de agosto de 2020 foram registrados 17 milhões de casos confirmados, com 680 mil mortes.

Períodos de epidemia são particularmente críticos para a saúde mental da população $0^{3-5}$. O medo que é uma reação instintiva e fundamental para o ser humano, muitas vezes se torna crônico ou desproporcional nesses períodos, contribuindo para o aumento das desordens psicológicas, incluindo a ansiedade ${ }^{6}$. Apesar dos dados conhecidos na população em geral $^{3-6}$, estudos sobre prevalência e fatores causais em grupos específicos, como os profissionais da saúde, são ainda escassos durante a pandemia causada pelo novo coronavirus $^{7-9}$.

Nas epidemias da síndrome respiratória aguda grave (SARS) em $2003^{10}$, H1N1 em 200911,12 e do Ebola em $2014^{13}$ estudos registraram elevada prevalência de ansiedade nos profissionais de saúde. Na epidemia da SARS, os estudos identificaram prevalência de ansiedade de $13 \%{ }^{14}$ a $51 \%{ }^{15}$. Durante a epidemia da Ebola, Ji et al. ${ }^{16}$ verificaram que $39 \%$ de profissionais de saúde apresentaram ansiedade.

Fatores como elevada carga horária de trabalho, estresse, pressão decorrente do elevado número de atendimento de casos graves, poucas horas de sono, infraestrutura inadequada, indisponibilidade de equipamentos de proteção individual em quantidade suficiente, o risco de ser infectado e de transmitir para familiares e outras pessoas podem contribuir para o aumento da ansiedade nesses períodos ${ }^{17-20}$.

Pappa et al. ${ }^{21}$ realizaram revisão sistemática sobre depressão, ansiedade e insônia, analisando a prevalência de ansiedade entre profissionais de saúde em 12 estudos. Entretanto, não analisaram a prevalência de ansiedade entre os profissionais da linha de frente, a associação entre o diagnóstico de COVID-19 e de doenças crônicas com a ansiedade, bem como não compararam a prevalência de ansiedade entre as diversas categorias profissionais.

É importante a realização de estudos que avaliem a prevalência de ansiedade nestes profissionais, de modo a desenvolver estratégias que visem favorecer a qualidade de vida e a saúde mental durante a pandemia da COVID-19 e, dessa forma, contribuir para a garantia da força de trabalho necessária para o combate a doença, bem como a saúde mental destes profissionais no período pós pandemia ${ }^{20,22-24}$.

Nesse contexto, o objetivo desta revisão sistemática com metanálise é identificar a prevalência de ansiedade em profissionais de saúde durante à pandemia da COVID-19.

\section{Métodos}

Revisão sistemática com metanálise elaborada e redigida conforme às recomendações do PRISMA (Preferred Reporting Items for Systematic Reviews and Meta-Analyzes) ${ }^{25}$.

\section{Pergunta de pesquisa}

Qual a prevalência de ansiedade nos profissionais da saúde durante a pandemia da COVID-19?

\section{Critérios de elegibilidade}

Foram considerados elegíveis estudos observacionais publicados em 2020 em qualquer idioma que apresentaram a prevalência de ansiedade em profissionais de saúde de qualquer faixa etária durante a pandemia da COVID-19.

$P$ - População: profissionais da saúde de qualquer faixa etária; I - Interesse: ansiedade; Co Contexto: pandemia da COVID-19.

Foram excluídos editoriais, artigos de revisão e estudos que não apresentaram a prevalência da ansiedade.

\section{Fontes de informação e estratégia de busca}

Foi realizada busca virtual nas bases de dados Embase, PubMed e LILACS em 11 de julho de 2020 por dois pesquisadores de forma independente. Além disso, foram analisadas as referências dos artigos selecionados para verificar se algum preenchia os critérios de inclusão, bem como busca manual. A estratégia de busca utilizada em cada base de dados é apresentada no Quadro 1.

\section{Triagem e seleção dos estudos}

O processo de seleção dos estudos para inclusão na revisão sistemática foi realizado inicialmente pela leitura do título e resumo por DS e revisado por FA e RC. Em seguida, DS e RC analisaram o texto-completo dos estudos selecionados para verificar o preenchimento dos critérios 
Quadro 1. Estratégia de busca e filtros utilizados por base de dados.

\begin{tabular}{|c|c|}
\hline & Estratégia de busca \\
\hline $\begin{array}{l}\text { Base de dados: } \\
\text { Embase. Busca } \\
\text { realizada em } 11 \\
\text { de julho de } 2020 . \\
\text { Filtro utilizado } \\
\text { - Período de } \\
\text { publicação: } \\
\text { 2019-2020. } \\
\text { Base de dados: } \\
\text { LILACS. Busca }\end{array}$ & 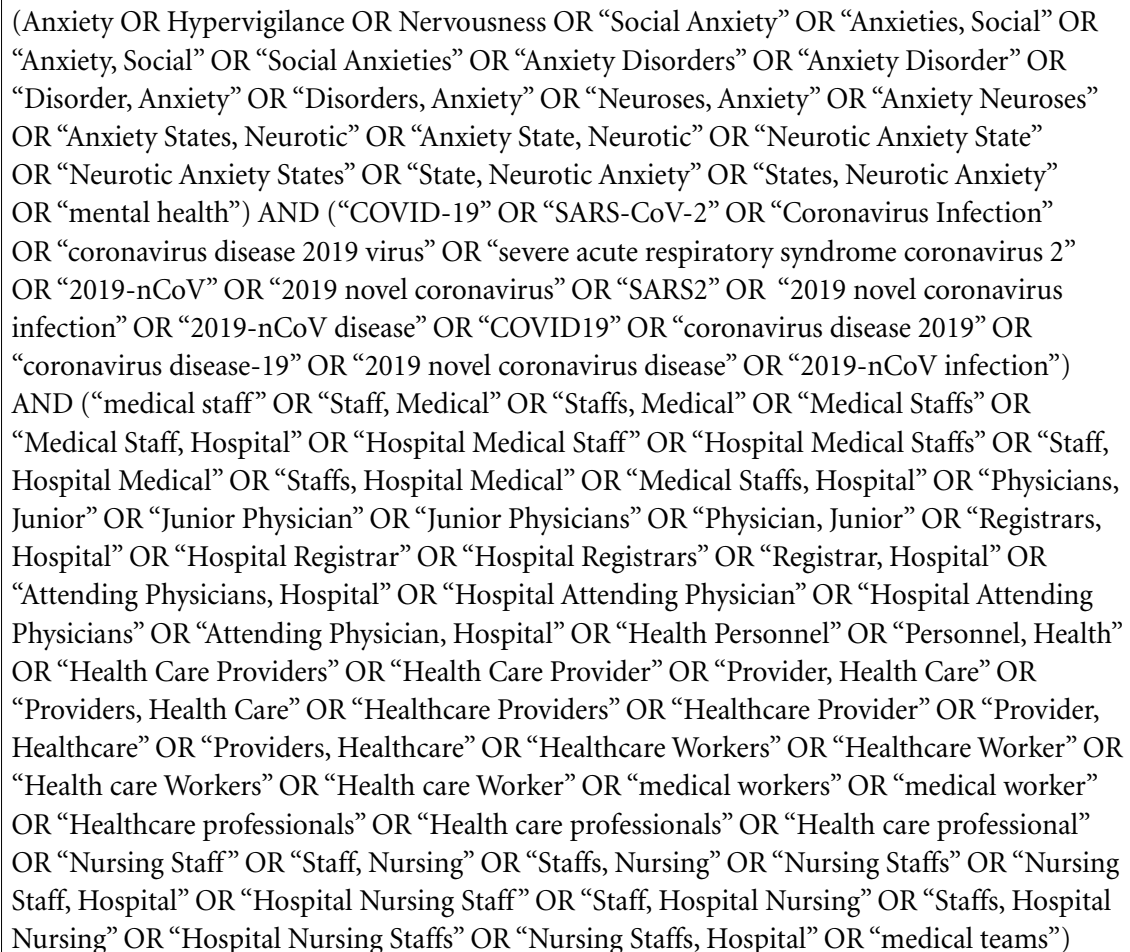 \\
\hline
\end{tabular}

de elegibilidade e, para os artigos em que houve conflito, a seleção foi revisada por FA.

\section{Extração dos dados}

Os dados coletados dos estudos foram: autoria, delineamento do estudo, número, sexo e faixa etária dos participantes, formação acadêmica, área de atuação profissional, instrumentos e critérios utilizados para a avaliação e diagnóstico da ansiedade e principais resultados obtidos.

\section{Avaliação da qualidade dos estudos}

A escala Newcastle-Ottawa adaptada para estudos transversais foi utilizada para a avaliação da qualidade dos estudos incluídos na revisão ${ }^{26}$. Este instrumento é composto por sete itens, nos domínios seleção (4 itens), comparação (1 item) e desfecho (2 itens). Porém, como nesta revisão não foi realizada análise sobre exposição e comparabilidade, foram considerados para análise cinco domínios: 1) representatividade da amos- tra; 2) tamanho da amostra; 3) taxa de resposta (não respondentes); 4) Avaliação do desfecho; e, 5) Análises estatísticas. Para cada item pode ser atribuída uma estrela, sendo que para desfecho pode se pontuar com até duas estrelas. Deste modo, a pontuação total pode ser de 0 a 6 pontos. Os estudos que receberam quatro estrelas ou mais foram classificados com boa qualidade metodológica.

\section{Análise dos dados}

O desfecho primário da metanálise foi a prevalência geral ( $\mathrm{p}$ ) de ansiedade nos profissionais de saúde, com o respectivo intervalo de confiança (IC) e a taxa percentual (p x 100\%), sendo incluídos os estudos com amostra igual ou maior que trinta $(\mathrm{n} \geq 30)$. O teste $\mathrm{Q}$ de Cochran e a estatística $\mathrm{I}^{2}$ foram utilizados para avaliar a heterogeneidade ${ }^{27,28}$. Foi estabelecido o emprego do modelo de efeitos aleatórios para a identificação da prevalência combinada quando verificada elevada heterogeneidade ( $\mathrm{I}^{2}>50 \%$ e teste Q 
de Cochran $P<0.10)$. No caso de heterogeneidade baixa ( $\mathrm{I}^{2}<50 \%$ e $\left.P>0.10\right)$, foi utilizado o modelo de efeitos fixos.

Foi realizada análise de subgrupo por categoria profissional, continente de atuação dos profissionais, pelos instrumentos empregados para o diagnóstico da ansiedade e qualidade metodológica dos estudos primários para explorar possíveis fontes de heterogeneidade. A estimativa do Odds Ratio (OR) com o respectivo intervalo de confiança (IC) para os desfechos dicotômicos sexo e profissão foi realizada utilizando modelos aleatórios $\left(\mathrm{I}^{2}>50 \%\right.$ e teste $\mathrm{Q}$ de Cochran $\left.P<0,10\right)$ ou modelos fixos $\left(\mathrm{I}^{2}<50 \%\right.$ e $\left.P>0,10\right)$. Análise de sensibilidade foi realizada retirando cada estudo e calculando a prevalência com os estudos remanescentes, com o objetivo de identificar quais afetaram consideravelmente a prevalência. A análise estatística foi realizada no software Revman ( $R e$ view Manager) versão 5.4 e no OpenMetaAnalyst.

Para os casos em que não foi possível conduzir metanálise para a avaliação da associação entre variáveis e a prevalência de ansiedade, foi empregada a síntese sem metanálise ${ }^{29}$. Para isso, foram considerados estudos com associação no mesmo sentido (por exemplo, associação positiva entre comorbidades e a prevalência de ansiedade) e comparados com estudos com resultados no sentido oposto (por exemplo, associação negativa entre comorbidades e a prevalência de ansiedade), ou que não identificaram associação significativa.

\section{Resultados}

A busca nas bases de dados recuperou 860 registros e um foi identificado por meio da busca manual, totalizando 861 artigos. Os duplicados ( $n=192)$ foram removidos, resultando em 669 estudos para avaliação. Depois da análise do título e resumo, 82 estudos tiveram o texto-completo avaliado. Trinta e seis estudos foram incluídos na revisão sistemática ${ }^{30-65}$. Na metanálise, foram incluídos 35 estudos com 32.367 participantes de 15 países em três continentes ${ }^{30-47,49-65}$. A Figura 1 apresenta o fluxograma de seleção dos estudos.

\section{Qualidade metodológica dos estudos}

Vinte e seis estudos incluídos na revisão apresentaram boa qualidade metodológi$\mathrm{Ca}^{30,34-36,38,41-47,49-51,53-58,60,62-65}$ e 10 foram classificados como tendo baixa qualidade $31-33,37,39,40,48,52,59,61$. A Tabela 1 apresenta a qualidade metodológica e as características dos estudos incluídos na revisão.

\section{Características dos estudos}

Todos os estudos incluídos na revisão sistemática foram de delineamento transversal ${ }^{30-65}$. Dezesseis estudos foram conduzidos na China $^{33,39,42-47,50,56,57,60,62-65}$, três na Itáliaa ${ }^{36,51,55}$, três no Irã $^{48,59,61}$, dois nos Estados Unidos ${ }^{35,52}$, um no Brasi $^{38}$, um na Jordânia ${ }^{49}$, um em Singapura ${ }^{53}$, um na Índia ${ }^{32}$, um na Coréia do Sul ${ }^{58}$, um na Irlanda ${ }^{37}$, um na Turquia ${ }^{40}$, um na Tailândia ${ }^{30}$, um no Nepal $^{41}$, um em Omã ${ }^{31}$, um na Arábia Saudita ${ }^{54}$ e um incluiu profissionais de Singapura e Índia ${ }^{34}$.

O número de participantes entre os estudos variou de $14^{48}$ a 5.062 profissionais de saúde ${ }^{64}$.Vinte e seis estudos incluíram mais mulheres que homens ${ }^{30,31,33,34,38-40,42-44,46,47,49-56,58,60-62,64,65}$, sendo que em 13 estudos as mulheres representaram mais de $75 \%$ dos participantes ${ }^{2,33,38,42-44,46,47,51,54,56,60,64}$. Os estudos incluíram profissionais de saúde com idade entre $18^{44,46,49,60}$ e mais que $75 \operatorname{anos}^{52}$.

Trinta e um estudos incluíram médi$\cos ^{30-35,37,39-41,43-57,59-62,64,65}, 29$ incluíram enfermei$\operatorname{ros}^{30-32,34,37-39,41-47,49-57,59-62,64,65}$, um incluiu farmacêuticos ${ }^{49}$, um incluiu fisioterapeutas ${ }^{58}$ e um incluiu dentistas ${ }^{36}$. Seis estudos apresentaram dados sobre o tempo de profissão ${ }^{42,43,44,57,60,64}$. Em todos os estudos o nível profissional mais frequente foi iniciante, seguido por intermediário e sênior ${ }^{42,43,44,57,60,64}$.

O número de profissionais que atuavam na linha de frente de combate a COVID-19 ou com maior risco de terem contato com pacientes com COVID-19 foi apresentado em 25 estudos $^{30-33,39-43,44-47,49,51-53,55-60,62,64,65}$. Em 12 estudos mais de $40 \%$ dos profissionais incluídos atuavam na linha de frente ou tiveram contato com

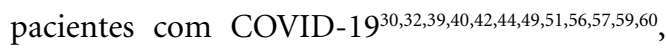
sendo que três estudos ${ }^{32,39,64}$ referem que $100 \%$ dos profissionais atuavam na linha de frente. $\mathrm{Lu}$ et al. ${ }^{47}$ verificaram que $20,4 \%$ dos profissionais apresentavam risco de contato com pacientes com COVID-19, no estudo de Chen et al. ${ }^{33}$ o percentual foi de $18,1 \%$, enquanto que no estudo de Zhang et al. ${ }^{62} 14,9 \%$ apresentavam elevado risco de contato com pacientes infectados pelo coronavírus. Wang et al. ${ }^{56}$ registraram que $62,6 \%$ já tinham atuado no tratamento de pacientes infectados pelo SARS-COV-2.

Sete estudos referiram o número de casos suspeitos ou confirmados de COVID-19 nos profissionais de saúde ${ }^{32,36,40,46,56,61,64}$. Neste sentido, Liu et al. ${ }^{46}$ verificaram que 41 participantes $(8 \%)$ eram casos suspeitos de COVID-19 e Chatterjee et al. ${ }^{32}$ referiram que $9,2 \%$ dos profissionais estavam em quarentena. No estudo de Zhang et. ${ }^{61}$ 2,3\% dos 


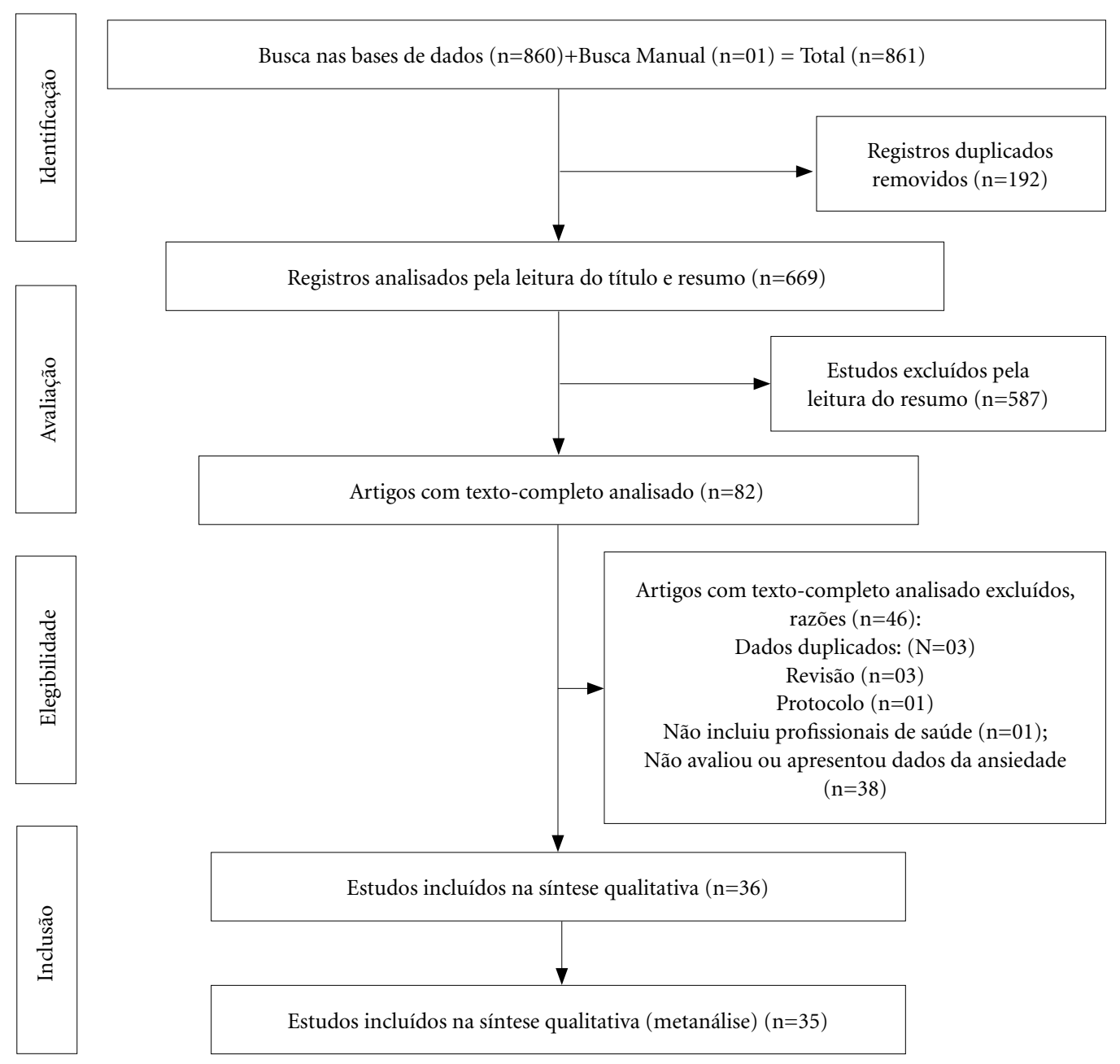

Figura 1. Fluxograma PRISMA de seleção dos estudos.

profissionais de saúde foram diagnosticados com COVID-19 e Zhu et al. ${ }^{64}$ registraram que 3,1\% eram casos suspeitos ou tiveram o diagnóstico de COVID-19.

O número de profissionais com doenças crônicas foi registrado em oito estudos ${ }^{32,34,37,40,41,53,62,64}$. Três estudos verificaram que menos de $20 \%$ dos profissionais apresentavam doenças crôni$\mathrm{cas}^{34,37,64}$. No estudo de Chatterjee et al. ${ }^{32}$ referiram que somente $5,9 \%$ não apresentavam doenças crônicas, sendo que 56,5\% apresentavam hipertensão arterial sistêmica, diabetes mellitus e doença pulmonar obstrutiva crônica.

\section{Critérios de avaliação da ansiedade}

Os estudos incluídos na revisão utilizaram diferentes critérios para a avaliação da ansiedade. A 7-item Generalized Anxiety Disorder (GAD-7) scal $^{66}$ foi utilizada para avaliar a ansiedade em 16 estudos $^{30,31,35,36,37,41,44,45,49,50,51,54,58,60,63,64}$. Seis estudos $^{32,34,40,53,5,59}$ utilizaram a Depression, anxiety and stress scales (DASS-21) ${ }^{67}$. A Zung Self-rating Anxiety Scale (SAS) ${ }^{68}$ foi utilizada para o diagnóstico da ansiedade em seis estudos ${ }^{33,42,43,46,56,64}$. Zhang et al..$^{62}$ e Shechter et al. ${ }^{52}$ avaliaram a ansiedade por meio da Generalized Anxiety Disorder 2-item (GAD-2) ${ }^{69}$. Zhang et al. ${ }^{61}$ referiram o em- 


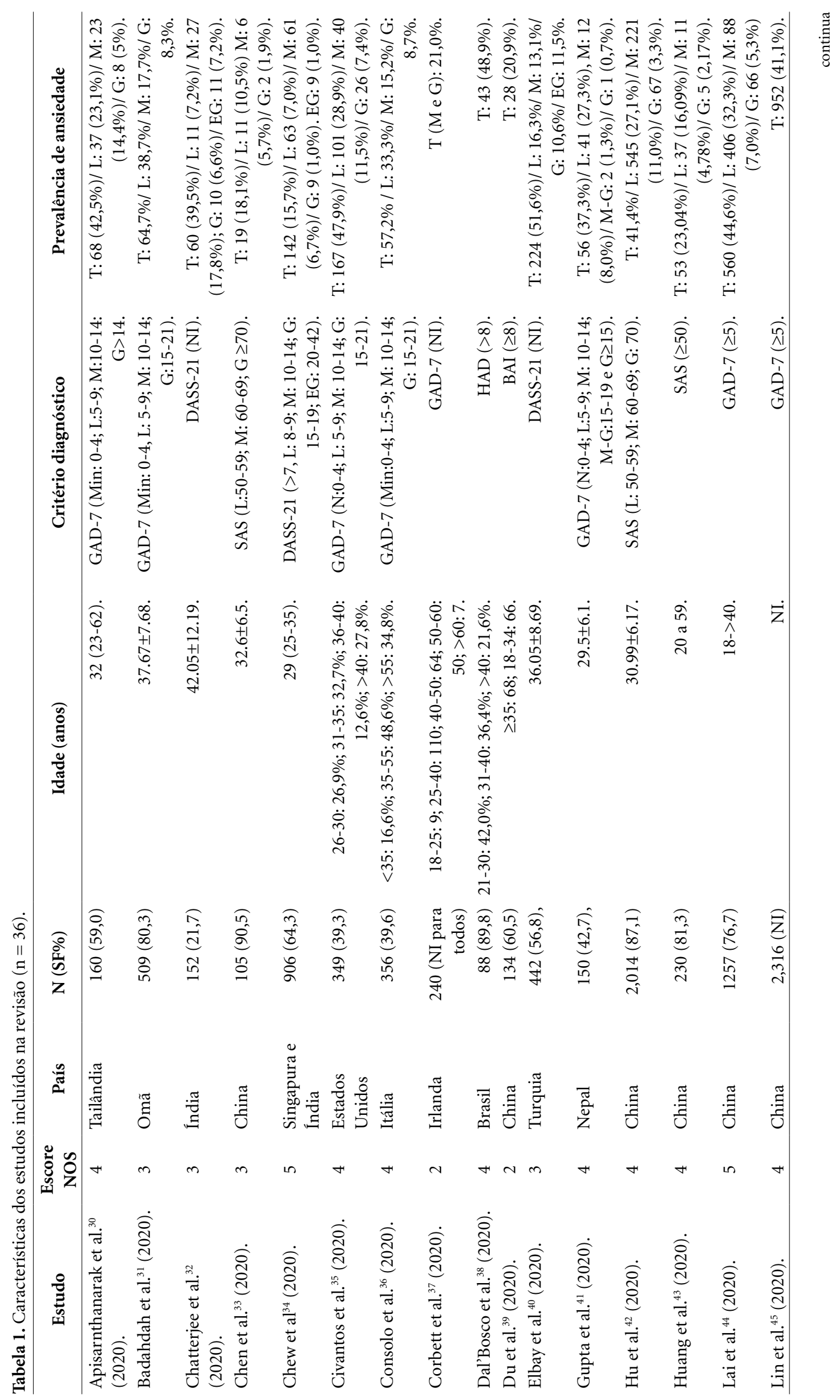




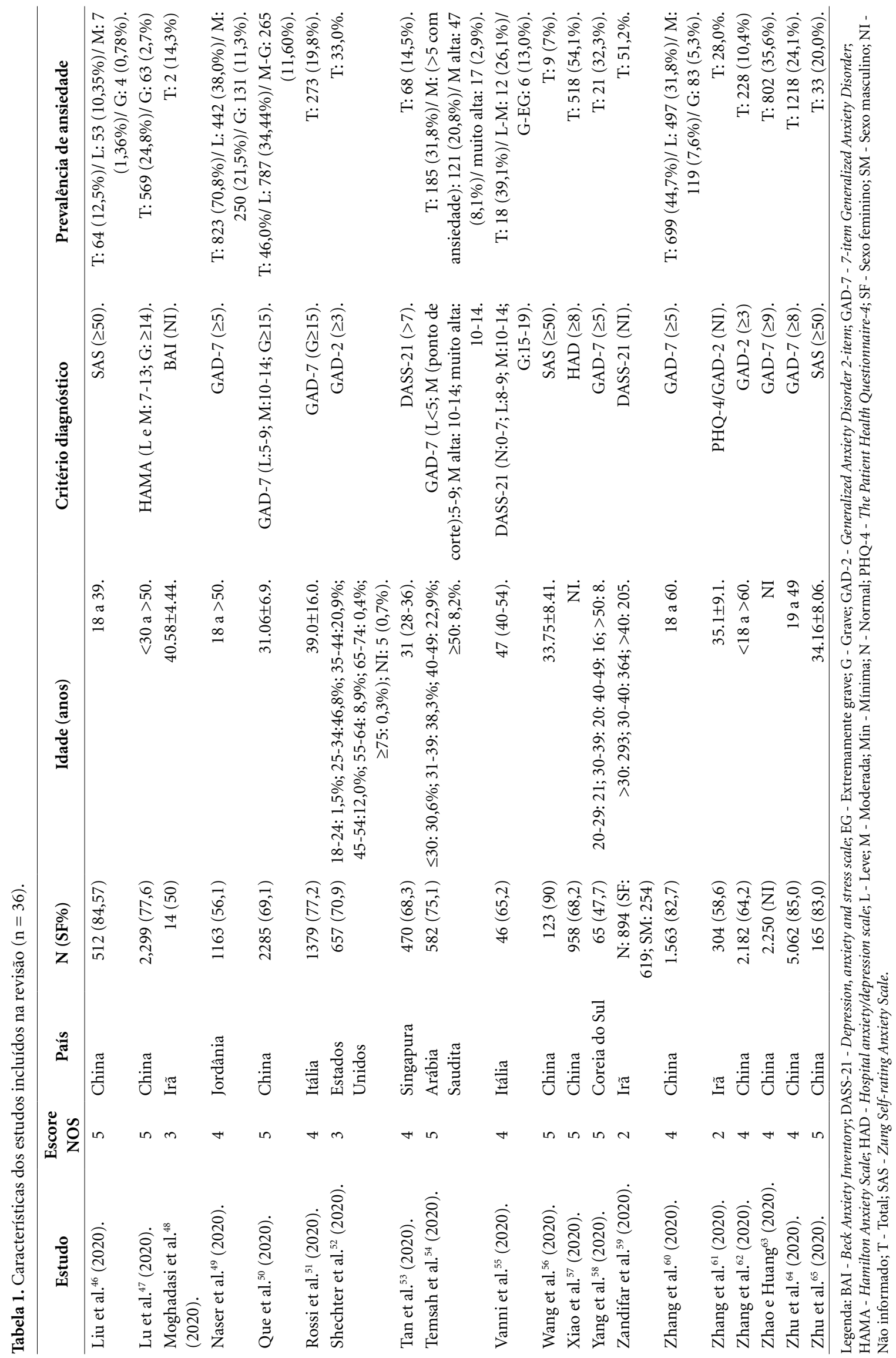


prego do Patient Health Questionnaire-4 (PHQ$4)^{70}$, que tem como subescala para avaliação da ansiedade o GAD-2.

$\mathrm{O}$ Beck Anxiety Inventory (BAI) ${ }^{71}$ foi utilizado em dois estudos ${ }^{39,48}$; a Hospital Anxiety and Depression Scale $(\mathrm{HAD})^{72}$ também foi empregada em dois estudos ${ }^{38,57}$; e no estudo realizado por Lu et al. ${ }^{47}$ foi utilizada a Hamilton Anxiety Scale (HAMA) $)^{73}$ para o diagnóstico da ansiedade. Os critérios dos instrumentos são apresentados no Quadro 2.

\section{Prevalência de ansiedade nos profissionais de saúde durante a pandemia da COVID-19}

A prevalência geral de ansiedade (leve, moderada e grave) foi de $35 \%$ (IC95\%: 29-40), variando de $7.0 \%{ }^{56}$ a $70.8 \%{ }^{49}$, com forte heterogeneidade entre os estudos $\left(\mathrm{I}^{2}=99 \%, P<0,01\right)$ (Figura 2). $\mathrm{Na}$ análise de sensibilidade removendo um estudo por vez foi verificado que os intervalos de confiança se sobrepõem em todos os casos analisados, de modo que nenhum estudo impactou significativamente na prevalência de ansiedade. No entan-

Quadro 2. Critérios de avaliação da ansiedade nos estudos incluídos na revisão.

\begin{tabular}{|c|c|c|}
\hline Instrumento & Critérios & Pontos de corte \\
\hline $\begin{array}{l}\text { 7-item } \\
\text { Generalized } \\
\text { Anxiety } \\
\text { Disorder (GAD- } \\
\text { 7) scale }\end{array}$ & $\begin{array}{l}\text { Este instrumento apresenta sete itens relacionados a sintomas de } \\
\text { ansiedade ocorridos nos últimos sete dias, com quatro opções de } \\
\text { resposta: } 0 \text { - de modo algum, } 1 \text { - vários dias, } 2 \text { - mais da metade } \\
\text { dos dias e } 3 \text { - quase todos os dias. Desse modo, a pontuação pode } \\
\text { variar de } 0 \text { a } 21 .\end{array}$ & $\begin{array}{l}\text { Sem ansiedade: } 0 \text { a } 4 \\
\text { pontos; ansiedade leve: } \\
5 \text { a } 9, \text { moderada: } 10 \text { a } 14, \\
\text { e grave: (15 a } 21) .\end{array}$ \\
\hline $\begin{array}{l}\text { Depression, } \\
\text { anxiety and } \\
\text { stress scales } \\
\text { (DASS-21) }\end{array}$ & $\begin{array}{l}\text { Este instrumento é um conjunto de três subescalas, cada uma com } \\
7 \text { itens, que avaliam depressão, ansiedade e estresse, conforme os } \\
\text { sintomas referidos na última semana antes da avaliação. A escala } \\
\text { é do tipo Likert, com quatro opções de resposta variando de } 0 \\
\text { (Não se aplicou de maneira alguma) a } 3 \text { (aplicou-se muito, ou na } \\
\text { maioria do tempo). Deste modo, a pontuação total é o somatório } \\
\text { das respostas de cada item, multiplicado por } 2\end{array}$ & $\begin{array}{l}\text { O ponto de corte é }>7, \\
\text { sendo de } 8-9 \text { (leve), } \\
10-14 \text { (moderada), } \\
15-19 \text { (grave) e } 20-42 \\
\text { (extremamente grave). }\end{array}$ \\
\hline $\begin{array}{l}\text { Zung Self-rating } \\
\text { Anxiety Scale } \\
(\text { SAS) }\end{array}$ & $\begin{array}{l}\text { A SAS, que avalia a frequência de sintomas de ansiedade nos } \\
\text { últimos dias, é composta por } 20 \text { itens com quatro opções de } \\
\text { resposta: } 1 \text { - pouco ou um pouco do tempo; } 2 \text { - algumas vezes; } \\
3 \text { - boa parte do tempo; e, } 4 \text { - na maioria ou todo o tempo. Cinco } \\
\text { itens do instrumento }(5,9,13,17 \text { e } 19) \text { recebem pontuação } \\
\text { invertida. Deste modo, a pontuação total varia de } 20 \text { a } 80\end{array}$ & $\begin{array}{l}<50 \text { pontos - normal; } 50 \\
\text { a } 60 \text { pontos - ansiedade } \\
\text { leve, } 61 \text { a } 70 \text { pontos - } \\
\text { ansiedade moderada; e, } \\
>70 \text { pontos - ansiedade } \\
\text { grave. }\end{array}$ \\
\hline \begin{tabular}{|l|} 
Generalized \\
Anxiety \\
Disorder 2-item \\
$($ GAD-2) \\
\end{tabular} & $\begin{array}{l}\text { Esse instrumento tem dois itens sobre a frequência dos sintomas } \\
\text { de ansiedade nos últimos sete dias com quatro opções de resposta: } \\
0 \text { - de modo algum, } 1 \text { - vários dias, } 2 \text { - mais da metade dos dias e } \\
3 \text { - quase todos os dias. O escore total varia de } 0 \text { a } 6 \text {. }\end{array}$ & Ansiedade: Escore $\geq 3$. \\
\hline $\begin{array}{l}\text { Patient Health } \\
\text { Questionnaire-4 } \\
\text { (PHQ-4) }\end{array}$ & $\begin{array}{l}\text { Este instrumento é composto pelas subescalas Patient Health } \\
\text { Questionnaire-2 (PHQ-2), utilizada para o diagnóstico da } \\
\text { depressão, e a GAD-2 para a ansiedade }\end{array}$ & Ansiedade: $\geq 3$ \\
\hline $\begin{array}{l}\text { Beck Anxiety } \\
\text { Inventory (BAI) }\end{array}$ & $\begin{array}{l}\text { O instrumento composto por } 21 \text { itens avalia sintomas de } \\
\text { ansiedade na última semana e tem quatro opções de resposta } \\
\text { variando de } 0 \text { (absolutamente não) a } 3 \text { (gravemente, dificilmente } \\
\text { pude suportar). A pontuação total varia de } 0 \text { a } 63 .\end{array}$ & Ansiedade: $>8$. \\
\hline $\begin{array}{l}\text { Hospital } \\
\text { Anxiety and } \\
\text { Depression } \\
\text { Scale (HAD) }\end{array}$ & $\begin{array}{l}\text { Cada uma das subescalas do instrumento (HAD-A para ansiedade } \\
\text { e HAD-D para depressão) tem sete questões referentes aos } \\
\text { sintomas dos últimos sete dias. Para cada pergunta pode ser } \\
\text { atribuída pontuação de } 0 \text { a } 3 \text {, com um máximo de até } 21 \text { pontos. }\end{array}$ & Ansiedade: escore $>8$. \\
\hline $\begin{array}{l}\text { Hamilton } \\
\text { Anxiety Scale } \\
\text { (HAMA) }\end{array}$ & $\begin{array}{l}\text { Esta escala tem } 14 \text { itens, com cinco opções de resposta, variando } \\
\text { de } 0 \text { (nunca) a } 4 \text { (muito grave). }\end{array}$ & $\begin{array}{l}\text { Sem ansiedade: } \\
0-6 ; \text { Ansiedade: } \geq 7 \\
\text { Ansiedade grave } \geq 14 \text {. }\end{array}$ \\
\hline
\end{tabular}




\section{Studies}

Apisarntharnarak et al. 2020

Badahdah et al. 2020

Chatterjee et al. 2020

Chen et al. 2020

Chew et al. 2020

Civantos et al. 2020

Consolo et al. 2020

Corbett et al. 2020

Dal'Bosco et al. 2020

Du et al. 2020

Elbay et al. 2020

Gupta et al. 2020

$\mathrm{Hu}$ et al. 2020

Huang J et al. 2020

Huang $\mathrm{N}$ et al. 2020

Lai et al. 2020

Liu C et al. 2020

Lu et al. 2020

Ma et al. 2020

Naser et al. 2020

Que et al. 2020

Rossi et al. 2020

Shechtera et al. 2020

Tan et al. 2020

Temsaha et al. 2020

Vanni et al. 2020

Wang et al. 2020

Xiao et al. 2020

Yang et al. 2020

Zandifar et al. 2020

Zhang C et al. 2020

Zhang S et al. 2020

Zhang W et al. 2020

Zhu et al. 2020

Zhu J et al. 2020

Overall $\left(I^{\wedge} 2=99,21 \%\right.$; P $\left.<0,001\right) \quad 0,347(0,292 ; 0,401)$

\section{Estimate (95\% C.I.)}

$0,435(0,348 ; 0,502)$

$0,647(0,605 ; 0,689)$

$0,395(0,317 ; 0,473)$

$0,181(0,107 ; 0,255)$

$0,157(0,133 ; 0,181)$

$0,479(0,427 ; 0,531)$

$0,572(0,521 ; 0,623)$

$0,210(0,158 ; 0,262)$

$0,489(0,385 ; 0,493)$

$0,209(0,140 ; 0,278)$

$0,516(0,469 ; 0,563)$

$0,373(0,296 ; 0,450)$

$0,230(0,176 ; 0,284)$

$0,356(0,336 ; 0,376)$

$0,446(0,419 ; 0,473)$

$0,125(0,096 ; 0,154)$

$0,248(0,239 ; 0,266)$

$0,411(0,391 ; 0,431)$

$0,708(0,682 ; 0,734)$

$0,469(0,440 ; 0,480)$

$0,198(0,177 ; 0,219)$

$0,330(0,294 ; 0,366)$

$0,145(0,113 ; 0,177)$

$0,318(0,280 ; 0,356)$

$0,391(0,250 ; 0,532)$

$0,541(0,509 ; 0,573)$

$0,323(0,209 ; 0,437)$

$0,512(0,479 ; 0,545)$

$0,447(0,422 ; 0,472)$

$0,280(0,229 ; 0,331)$

$0,104(0,091 ; 0,117)$

$0,241(0,229 ; 0,253)$

$0,200(0,139 ; 0,261)$
$0,414(0,392 ; 0,436)$

$0,070(0,025 ; 0,115)$

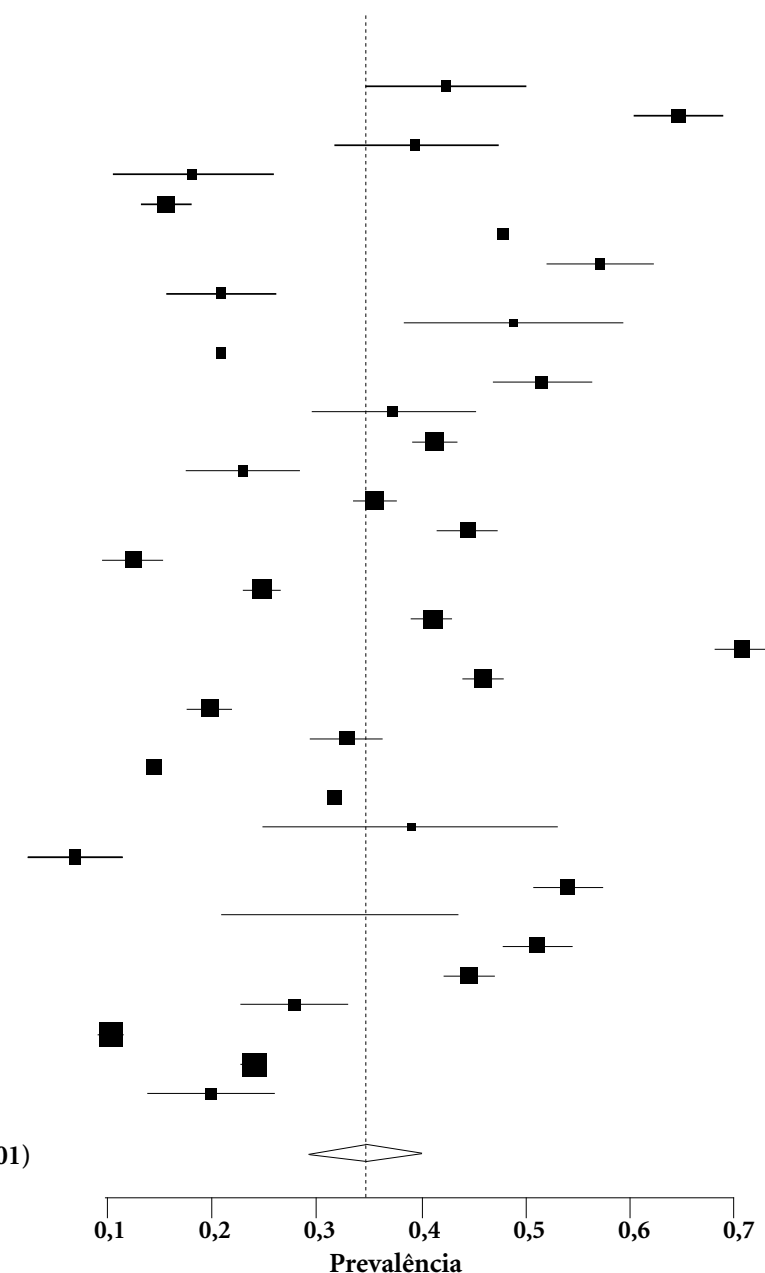

Figura 2. Prevalência de ansiedade em profissionais de saúde durante a pandemia da COVID-19.

Legenda: Studies - Estudos; Estimate - Estimativa; 95\% C.I. - 95\%IC (intervalo de confiança); Overall - Total.

to, pela inspeção visual, se observa considerável alteração nos intervalos de confiança com a remoção dos estudos de Badahdah et al. ${ }^{31}$, Naser et al. ${ }^{49}$, Wang et al. ${ }^{56}$ e Zhang et al. ${ }^{62}$, sugerindo que estes estudos podem ser os que mais contribuíram para a elevada heterogeneidade observada (Figura 4). Foi identificada diferença significativa na prevalência com base nos instrumentos utilizados para o diagnóstico da ansiedade $(P<0,01)$. Na comparação da prevalência entre os continentes Ásia, Europa e América, não foi identificada diferença $(P=0,42)$. Não foi registrada diferença na prevalência de ansiedade na comparação entre os estudos com boa e baixa qualidade metodológica $(P=0,69)$. A Tabela 2 apresenta a prevalência de ansiedade por análise de subgrupo.

$\mathrm{Na}$ análise por sexo, realizada em 12 estudos, foi registrado maior risco de ansiedade nas mulheres que nos homens (Odds ratio [OR]: 1,64 [IC95\%: 1,47-1,84], $P<0.01$ ) (Figura 3A). Com relação a profissão, foi verificado maior risco de ansiedade entre enfermeiros em relação aos médicos (OR: 1,19 [IC95\%: 1,07-1,33] $P<0.01$ ) (Figura 3B). Na análise de subgrupo para preva- 


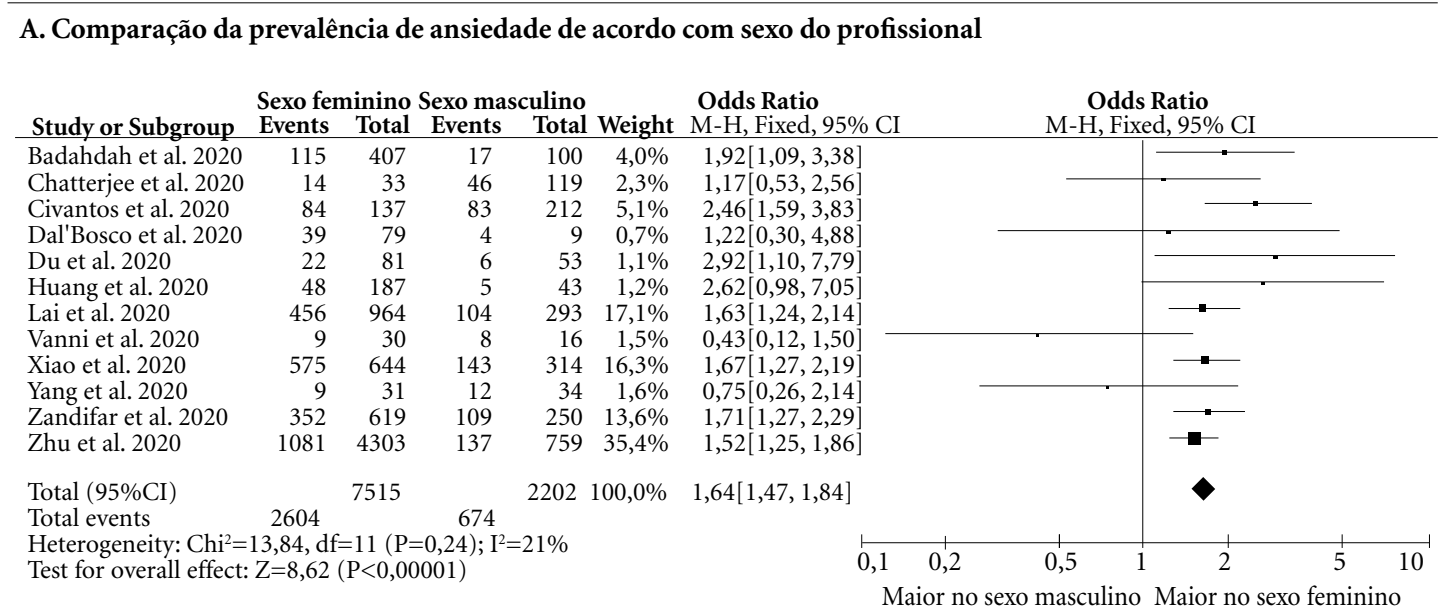

B. Comparação da prevalência de ansiedade entre enfermeiros e médicos durante a pandemia da COVID-19.

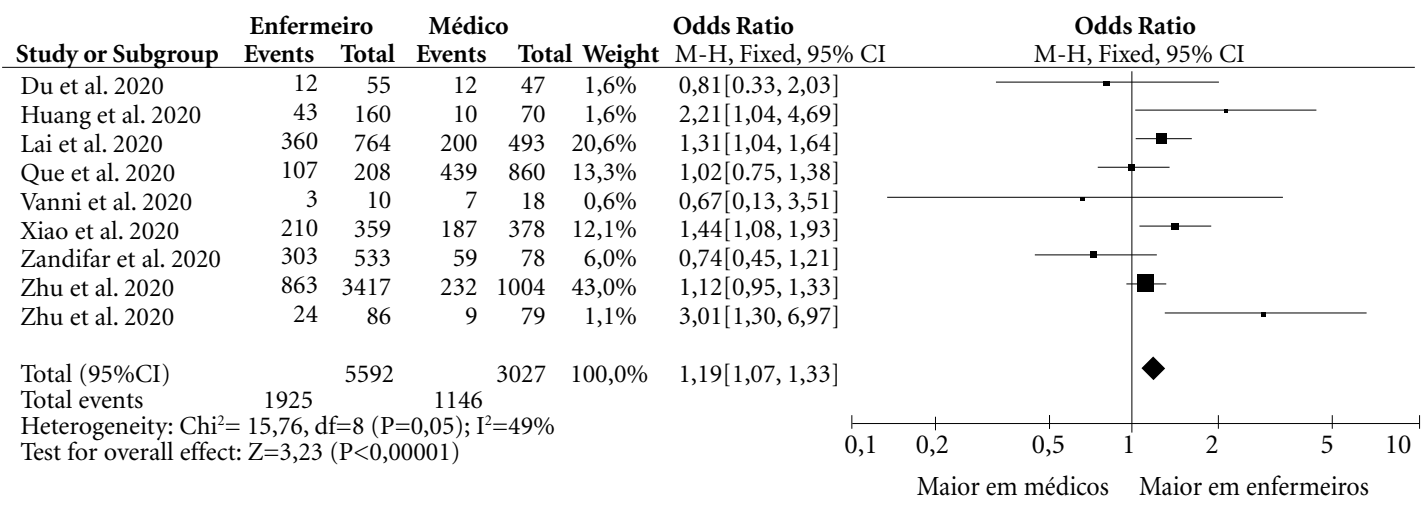

Figura 3. Prevalência da ansiedade por sexo e formação dos profissionais da saúde durante a pandemia da Covid-19.

Legenda: Study or Subgroup - Estudo ou Subgrupo; Events - Eventos; Weight - Peso; M-H, Fixed - Modelo de efeito fixo; 95\% CI - 95\% IC (intervalo de confiança); Total events - Total de eventos; Heterogeinity - Heterogeneidade; Test for overall effect - Teste para efeito total.

lência de ansiedade nos diferentes níveis profissionais (iniciante, intermediário e sênior) não foi identificada diferença $(P=0,60)$ (Tabela 2).

Sete estudos ${ }^{40,44,46,47,50,57,62}$ verificaram que os profissionais que atuavam na linha de frente no combate a COVID-19 ou com maior risco de ter contato com pacientes diagnosticados com COVID-19 apresentaram maior prevalência de ansiedade na comparação com os que não atuavam na linha de frente ou com menor risco de contato. No entanto, outros seis estudos não verificaram diferença entre os grupos ${ }^{31,33,51,55,59,64}$.

Liu et al. ${ }^{46}$ verificaram que os profissionais de saúde com suspeita de COVID-19 apresentavam maior risco de ansiedade que os profissionais que não eram casos suspeitos (escore SAS, casos suspeitos: média de 42,52, com desvio padrão [DP] de 9,69 versus não casos suspeitos: média 39,05, com DP de 8,62). Zhu et al. ${ }^{64}$ identificaram maior prevalência de casos de COVID-19 entre os profissionais com ansiedade (4,6\%) que entre profissionais que não apresentavam ansiedade (2,7\%). Zhang et al. ${ }^{61}$ não identificaram associação entre o diagnóstico de COVID-19 com ansiedade (Coeficiente de regressão [IC95\%]: 0,157; -0,956$1,271)$, mas, verificaram associação entre incerteza sobre estar infectado e ansiedade ( [IC95\%]: 0,688; 0,303-1,073).

A associação entre ansiedade e doenças crônicas preexistentes foi avaliada em três estudos ${ }^{32,62,64}$. 
Studies

Overall

Apisarntharnarak et al. 2020

Badahdah et al. 2020

Chatterjee et al. 2020

Chen et al. 2020

Chew et al. 2020

Civantos et al. 2020

Consolo et al. 2020

Corbett et al. 2020

Dal'Bosco et al. 2020

Du et al. 2020

Elbay et al. 2020

Gupta et al. 2020

Hu et al. 2020

Huang J et al. 2020

Huang N et al. 2020

Lai et al. 2020

Liu C et al. 2020

Lu et al. 2020

Ma et al. 2020

Naser et al. 2020

Que et al. 2020

Rossi et al. 2020

Shechtera et al. 2020

Tan et al. 2020

Temsaha et al. 2020

Vanni et al. 2020

Wang et al. 2020

Xiao et al. 2020

Yang et al. 2020

Zandifar et al. 2020

Zhang C et al. 2020

Zhang S et al. 2020

Zhang W et al. 2020

Zhu et al. 2020

Zhu J et al. 2020
Estimate (95\% C.I.)

$0,397(0,292 ; 0,401)$

$0,345(0,289 ; 0,400)$ $0,338(0,284 ; 0,392)$

$0,345(0,290 ; 0,401)$

$0,352(0,296 ; 0,407)$

$0,353(0,297 ; 0,408)$

$0,343(0,288 ; 0,396)$

$0,340(0,285 ; 0,395)$

$0,351(0,295 ; 0,406)$

$0,343(0,288 ; 0,398)$

$0,351(0,295 ; 0,406)$

$0,352(0,287 ; 0,397)$

$0,346(0,291 ; 0,401)$

$0,345(0,289 ; 0,401)$

$0,350(0,295 ; 0,406)$

$0,347(0,290 ; 0,403)$

$0,344(0,288 ; 0,399)$

$0,353(0,298 ; 0,409)$

$0,350(0,293 ; 0,407)$

$0,345(0,289 ; 0,401)$

$0,336(0,286 ; 0,386)$

$0,343(0,288 ; 0,399)$

$0,351(0,295 ; 0,408)$

$0,347(0,291 ; 0,403)$

$0,353(0,297 ; 0,408)$

$0,348(0,292 ; 0,403)$

$0,346(0,290 ; 0,401)$

$0,355(0,300 ; 0,410)$

$0,341(0,286 ; 0,396)$

$0,347(0,292 ; 0,403)$

$0,342(0,287 ; 0,397)$

$0,344(0,288 ; 0,399)$

$0,349(0,293 ; 0,404)$

$0,354(0,303 ; 0,405)$

$0,350(0,291 ; 0,409)$

$0,351(0,296 ; 0,407)$

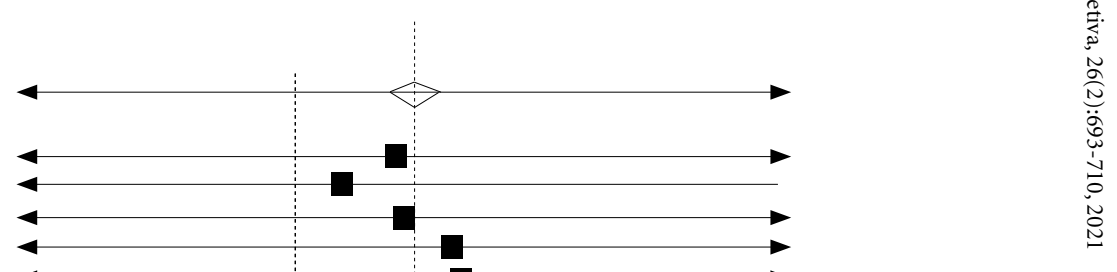

Figura 4. Gráfico da análise de sensibilidade removendo um estudo por vez.

Legenda: Studies - Estudos; Estimate - Estimativa; 95\% C.I. - 95\%IC (intervalo de confiança); Overall - Total.

Zhu et al. ${ }^{64}$ verificaram que $23,1 \%$ dos profissionais com diagnóstico de ansiedade apresentavam doenças crônicas, enquanto entre os profissionais sem ansiedade 14,8\% referiram ter alguma doença crônica. No estudo de Zhang et al. ${ }^{62}$ também relataram maior risco de ansiedade nos profissionais com doenças preexistentes (OR: 2,85; IC95\%: 1,73-4,68). No entanto, Chatterjee et al. ${ }^{32}$ identificaram prevalência de 55,6\% nos profissionais que não tinham nenhuma comorbidade, enquanto que nos que tinham diabetes mellitus, hipertensão e doença pulmonar obstrutiva crônica a prevalência foi de 36,1\%. Como neste estudo de Chatterjee et al. ${ }^{32}$ somente nove $(5,9 \%)$ profissionais não apresentaram comorbidades, se observa necessidade de maior cautela em sua análise.

\section{Discussão}

Nesta revisão sistemática, os resultados indicam elevada prevalência de ansiedade nos profissionais da saúde durante a pandemia da COVID-19, evidenciando a necessidade de medidas que vi- 
Tabela 2. Prevalência de ansiedade em profissionais de saúde durante a pandemia da COVID-19 de acordo com instrumento utilizado, continentes de atuação e categoria profissional.

\begin{tabular}{|c|c|c|c|}
\hline Análises de subgrupos & Número de estudos & $\begin{array}{c}\text { Prevalência geral } \\
\text { (IC95\%) }\end{array}$ & Teste estatístico (Valor de p) \\
\hline Critério diagnóstico & & & $\mathrm{Chi}^{2}=283,47, \mathrm{df}=6(\mathrm{P}<0,0001)$ \\
\hline GAD-7 & 16 & $41(44-49)$ & \\
\hline GAD-2 & 3 & $24(7-40)$ & \\
\hline SAS & 6 & $21(11-31)$ & \\
\hline DASS-21 & 6 & $35(19-51)$ & \\
\hline HAMA & 1 & $25(23-27)$ & \\
\hline BAI & 1 & $21(14-28)$ & \\
\hline HAD & 2 & $54(51-57)$ & \\
\hline Continente & & & $\mathrm{Chi}^{2}=1,75, \mathrm{df}=2(\mathrm{P}=0,42)$ \\
\hline Ásia & 26 & $34(28-40)$ & \\
\hline Europa & 4 & $34(15-53)$ & \\
\hline América & 3 & $43(31-55)$ & \\
\hline Categoria profissional & & & $\mathrm{Chi}^{2}=1,01, \mathrm{df}=2(\mathrm{P}=0,60)$ \\
\hline Iniciante & 4 & $30(17-43)$ & \\
\hline Intermediário & 4 & $39(24-54)$ & \\
\hline Sênior & 4 & $30(21-39)$ & \\
\hline Qualidade metodológica & & & $\mathrm{Chi}^{2}=0,16, \mathrm{df}=1(\mathrm{P}=0,69)$ \\
\hline Boa qualidade & 26 & $34(28-40)$ & \\
\hline Baixa qualidade & 9 & $37(26-47)$ & \\
\hline
\end{tabular}

sem prevenir este transtorno e que resultem em melhorias na qualidade de vida e na saúde psicológica destes trabalhadores. Além disso, não foi verificada diferença na prevalência entre os continentes, evidenciando que a doença causada pelo novo coronavírus tornou a ansiedade prevalente entre profissionais de saúde nas mais diversas regiões do mundo.

Os estudos incluídos na revisão utilizaram diferentes instrumentos para o diagnóstico da ansiedade. Houve diferença significativa na prevalência de ansiedade conforme o instrumento utilizado. Estas diferenças podem ser explicadas considerando que os diversos instrumentos utilizados nos estudos foram desenvolvidos para público e contexto específicos, bem como apresentam diferentes sensibilidade e especificidade ${ }^{74}$.

A prevalência de ansiedade identificada nesta metanálise para os profissionais de saúde durante a pandemia da COVID-19 é semelhante a identificada na população em geral neste mesmo período. Em metanálise que incluiu 17 estudos, Salari et al. ${ }^{75}$ identificaram prevalência de ansiedade na população geral durante a pandemia da COVID-19 de 31,9\% (IC95\%: 27,5-36,7).
Vários fatores foram relacionados com o maior risco para ansiedade, os quais devem ser considerados no processo de tomada de decisão quanto às estratégias e grupos prioritários para as ações de prevenção e tratamento da ansiedade nos profissionais de saúde. As mulheres apresentaram maior risco de ansiedade que os homens. Esta diferença na prevalência de ansiedade entre os sexos tem sido referida por vários autores e registrada em estudos com a população em geral $^{76,77}$. Dados do Global Burden of Disease Study ${ }^{78}$ de 2015 registraram prevalência global de ansiedade nas mulheres de $4,6 \%$ e nos homens de 2,6\%. Neste estudo ${ }^{78}$, maior prevalência de ansiedade no sexo feminino em relação ao sexo masculino foi identificada em todas as faixas etárias analisadas, bem como em todos os continentes.

Além do possível papel dos hormônios sexuais estradiol e progesterona no desenvolvimento da ansiedade ${ }^{79}$, uma das teorias da área psicossocial sobre o maior risco de ansiedade relacionada ao gênero, a self-construal theory, refere que mulheres e homens se interpretam de maneira diferente ${ }^{80}$. Neste sentido, enquanto os homens analisam a si próprios de maneira independente aos 
outros, as mulheres tendem a se analisar relacionando com os demais, de modo interdependente.

Além disso, o fato de as mulheres representarem em maior proporção a força de trabalho em saúde - aproximadamente $80 \%$, inclusive atuando na linha de frente ${ }^{81,82}$, pode explicar o fato de estarem mais sujeitas a apresentarem ansiedade que os homens.

Com relação a profissão, houve risco significativamente maior de ansiedade nos enfermeiros, na comparação com médicos. Considerando que há a possibilidade de haver mais mulheres que homens entre os profissionais de enfermagem, este resultado pode ser fruto de confundimento por sexo, pois foi registrado maior risco de ansiedade nas mulheres que nos homens ${ }^{31,35,39,43,44,57,59,65}$. O maior tempo de cuidado direto com pacientes da equipe de enfermagem em comparação aos médicos pode ser uma das explicações para o maior risco de ansiedade em enfermeiros registrada em alguns estudos ${ }^{83}$. Neste sentido, Butler et al. ${ }^{84}$ verificaram que em $13,1 \%$ do tempo os pacientes recebem cuidados dos médicos, enquanto para os enfermeiros o percentual é de $86,1 \%$. Ademais, como os enfermeiros passam mais tempo com os pacientes, bem como geralmente são os profissionais que realizam a coleta de secreções das vias aéreas ou de sangue para os exames que detectam SARS-CoV-2 ou anticorpos, podem estar mais expostos a infecções que os médicos, contribuindo para a maior ansiedade nestes profissionais ${ }^{85,86}$.

A prevalência de ansiedade entre os profissionais com diferentes graus de experiência não se mostrou mais elevada entre os iniciantes. Considerando que a pandemia da COVID-19 é um momento que requer maior cuidado e perícia na oferta de serviços em saúde, se entende que os profissionais de nível intermediário e sênior, que apresentam maior experiência e expertise que os iniciantes, podem estar mais sobrecarregados com a elevada demanda de atendimento ${ }^{86,87}$. No caso dos profissionais iniciantes, por terem menos experiência, podem apresentar maior insegurança ${ }^{87}$. Ademais, neste período de enfrentamento a COVID-19, muitos profissionais de saúde recémformados foram recrutados para o atendimento clínico durante a pandemia. O início no primeiro emprego neste período pode também representar um dos fatores contribuintes para a ansiedade nos profissionais iniciantes.

Os resultados, na maioria dos estudos incluídos nesta revisão, apontaram maior prevalência de ansiedade nos profissionais atuantes na linha de frente quando comparados com aqueles menos expostos aos cuidados de pacientes infectados. Vários estudos ${ }^{40,44,46,47,50,57,62}$ registraram maior prevalência de ansiedade entre os profissionais que atuavam na linha de frente no combate a COVID-19. Dentre os fatores que podem explicar maior prevalência nestes profissionais se tem a preocupação em fornecer efetivo e humanizado atendimento a elevado número de pacientes críticos; o maior número de encontros profissional-paciente, o maior tempo em cada encontro com os pacientes, a maior proximidade física com os pacientes; o não recebimento de equipamentos de proteção individual em quantidade e qualidade adequadas para o período de trabalho e o medo de ser infectado pelo SARS-COV-2 e de transmitir o vírus para os familiares $^{88,89}$.

Estudos realizados durante outras epidemias também revelaram elevada prevalência de estresse e ansiedade em profissionais de saúde com maior risco de contato ou em contato direto com pacientes infectados ${ }^{90,91}$. Revisão sistemática realizada por Brooks et al. ${ }^{91}$ demonstrou maior prevalência de ansiedade e esgotamento físico em profissionais que atuaram na linha de frente durante epidemias, inclusive na SARS em 2003.

Os profissionais que referiram ser casos suspeitos ou que foram diagnosticados com a COVID-19 apresentaram mais ansiedade que os não infectados, conforme também registrado na população em geral, tanto em outras epidemias ${ }^{11,12}$, quanto na pandemia da COVID-19. Kong et al. ${ }^{92}$ identificaram que $34,7 \%$ dos pacientes com COVID-19 apresentaram ansiedade. $\mathrm{O}$ medo de transmitir para os familiares e o maior risco à saúde em decorrência da doença são possíveis fatores que podem explicar a maior ansiedade nestes profissionais ${ }^{88}$.

Dois estudos verificaram que os profissionais de saúde com doenças crônicas apresentaram maior prevalência de ansiedade ${ }^{62,64}$. O maior risco de complicações e morte relacionadas à COVID-19 em pessoas com doenças crônicas são fatores que explicam a maior ansiedade nesse caso $^{88}$.

Esse estudo apresenta como ponto positivo a avaliação da prevalência e fatores associados à ansiedade em profissionais de saúde durante à pandemia da COVID-19, podendo contribuir para o processo de tomada de decisão quanto aos grupos prioritários para direcionamento das ações de prevenção e tratamento. Entre as limitações destaca-se o não registro do protocolo da revisão; no entanto, a estratégia de busca completa para cada base de dados com os filtros aplicados foi disponibilizada de modo a proporcionar a reprodutibilidade dos achados. Além disso, foi 
registrada elevada heterogeneidade entre os estudos incluídos na revisão, pois nos estudos os autores utilizaram diferentes escalas e pontos de corte para a avaliação e o diagnóstico da ansiedade. Ademais, muitos tinham informações incompletas sobre o diagnóstico da COVID-19 por subgrupos, comprometendo as análises e rebaixando o nível da evidência encontrada. Ademais, os estudos foram conduzidos com diferentes amostras populacionais e em diferentes regiões do planeta em que condições de trabalho, acesso a equipamentos de proteção individual e capacidade técnica dos profissionais podem variar, resultando em maior ou menor prevalência de ansiedade.

\section{Contribuições do estudo e implicações para a prática clínica}

Esta revisão sistemática evidencia que a prevalência de ansiedade em profissionais de saúde durante a pandemia da COVID-19 é alta e maior entre as mulheres, profissionais de enfermagem e nos que atuam na linha de frente. Deste modo, estes resultados podem contribuir para o processo de tomada de decisão quanto aos grupos prioritários para as ações de prevenção e tratamento da ansiedade. Entretanto, limitações desta revisão como predomínio de estudos envolvendo médicos e profissionais da enfermagem, impedem que os resultados sejam extrapolados para outras profissões da saúde que também atuam na linha de frente no combate a COVID-19, como os fisioterapeutas. Por outro lado, ao apresentar dados comparando prevalência de ansiedade entre médicos e enfermeiros, este estudo aponta um fator potencial de afastamento do trabalho por motivo de saúde destes profissionais, com implicações importantes para a prática clínica, como por exemplo redução de médicos e profissionais de enfermagem durante a pandemia, com risco de assistência inadequada nos serviços de saúde públicos e privados.

\section{Conclusão}

A prevalência de ansiedade nos profissionais de saúde durante à pandemia da COVID-19 é elevada, independentemente do continente de atuação profissional e da qualidade metodológica dos estudos. A ansiedade foi mais prevalente nos profissionais do sexo feminino, enfermeiros, que atuam na linha de frente, infectados pelo SAR$\mathrm{S}-\mathrm{CoV}-2$ e que apresentavam doenças crônicas. Há escassez de estudos nos países com maior número de casos como Brasil e Estados Unidos e entre profissionais com importante atuação na pandemia como fisioterapeutas. Medidas que visem a prevenção e o tratamento da ansiedade nos profissionais de saúde que atuam no combate a COVID-19, bem como o fornecimento de seguimento e suporte adequados para os com ansiedade moderada e grave, são necessárias para melhorar a qualidade de vida e para garantir a força de trabalho fundamental para o enfrentamento da pandemia, bem como a saúde mental destes profissionais no período pós-pandemia. 


\section{Colaboradores}

Concepção, planejamento, coleta, análise, interpretação dos dados e redação do trabalho: DFO Silva, RN Cobucci e FB Andrade. Interpretação dos resultados e redação ou revisão do manuscrito: VP Soares-Rachetti e SCVC Lima. Todos os autores aprovaram a versão final encaminhada.

\section{Referências}

1. Lai CC, Shih TP, Ko WC, Tang HJ, Hsueh PR. Severe acute respiratory syndrome coronavirus 2 (SARSCoV-2) and corona virus disease-2019 (COVID-19): the epidemic and the challenges. Int J Antimicrob Agents 2020; 55(3):105924.

2. World Health Organization (WHO). Coronavirus disease 2019 (COVID-19). Situation Report - 195 [Internet]. 2020 [acessado 2020 Ago 3]. Disponível em: https: https://www.who.int/docs/default-source/coronaviruse/situation-reports/20200802-covid-19-sitrep-195.pdf?sfvrsn $=5 \mathrm{e} 5 \mathrm{da} 0 \mathrm{c5} \_2$

3. Ahmed MZ, Ahmed O, Aibao Z, Hanbin S, Siyu L, Ahmad A. Epidemic of COVID-19 in China and associated Psychological Problems. Asian J Psychiatr 2020; 51:102092.

4. Rajkumar RP. COVID-19 and mental health: A review of the existing literature. Asian J Psychiatr 2020; 52:102066.

5. Gao J, Zheng P, Jia Y, Chen H, Mao Y, Chen S, Wang Y, Fu H, Dai J. Mental health problems and social media exposure during COVID-19 outbreak. Plos One 2020; 15(4):e0231924.

6. Ornell, Schuch JB, Sordi AO, Kessler FHP. "Pandemic fear" and COVID-19: mental health burden and strategies. Braz J Psychiatry 2020; 42(3):232-235.

7. Lee SM, Kang WS, Cho AR, Kim T, Park JK. Psychological impact of the 2015 MERS outbreak on hospital workers and quarantined hemodialysis patients. Compr Psychiatry 2018; 87:123-127.

8. Gee S, Skovdal M. Navigating 'riskscapes': The experiences of international health care workers responding to the Ebola outbreak in West Africa. Health Place 2017; 45:173-180.

9. World Health Organization (WHO). Mental health and psychosocial considerations during the COVID-19 outbreak [Internet]. 2020 [acessado 2020 Abr 12]. Disponível em: https://apps.who.int/iris/bitstream/ handle/10665/331490/WHO-2019-nCoV-MentalHealth-2020.1-eng.pdf

10. Chan AO, Huak CY. Psychological impact of the 2003 severe acute respiratory syndrome outbreak on health care workers in a medium size regional general hospital in Singapore. Occup Med 2004; 54(3):190-196.

11. Everts J. Announcing swine flu and the interpretation of pandemic anxiety. Antipode 2013; 45(4):809-825.

12. Taha S, Matheson K, Cronin T, Anisman H. Intolerance of uncertainty, appraisals, coping, and anxiety: The case of the $2009 \mathrm{H} 1 \mathrm{~N} 1$ pandemic. Br J Health Psychol 2014; 19(3):592-605.

13. Lehmann M, Bruenahl CA, Löwe B, Addo MM, Schmiedel S, Lohse AW, Schramm C. Ebola and psychological stress of health care professionals. Emerg Infect Dis 2015; 21(5):913.

14. Bai Y, Lin CC, Lin CY, Chen JY, Chue CM, Chou P. Survey of stress reactions among health care workers involved with the SARS outbreak. Psychiatr Serv 2004; 55(9):1055-1057.

15. Wong WC, Wong SY, Lee A, Goggins WB. How to provide an effective primary health care in fighting against severe acute respiratory syndrome: the experiences of two cities. Am J Infect Control 2007; 35(1):50 55 . 
16. Ji D, Ji YJ, Duan XZ, Li WG, Sun ZQ, Song XA, Meng YH, Tang HM, Chu F, Niu XX, Chen GF. Prevalence of psychological symptoms among Ebola survivors and healthcare workers during the 2014-2015 Ebola outbreak in Sierra Leone: a cross-sectional study. Oncotarget 2017; 8(8):12784.

17. Park JS, Lee EH, Park NR, Choi YH. Mental health of nurses working at a government-designated hospital during a MERS-CoV outbreak: a cross-sectional study. Arch Psychiatr Nurs 2018; 32(1):2-6.

18. Vyas KJ, Delaney EM, Webb-Murphy JA, Johnston SL. Psychological impact of deploying in support of the US response to Ebola: a systematic review and meta-analysis of past outbreaks. Mil Med 2016; 181(11-12):e1515-e1531.

19. Ornell F, Halpern SC, Kessler FHP, Narvaez JCM. The impact of the COVID-19 pandemic on the mental health of healthcare professionals. Cad Saude Publica 2020; 36(4):e00063520.

20. Greenberg N. Mental health of health-care workers in the COVID-19 era. Nat Rev Nephrol 2020; 16:425-426.

21. Pappa S, Ntella V, Giannakas T, Giannakoulis VG, Papoutsi E, Katsaounou P. Prevalence of depression, anxiety, and insomnia among healthcare workers during the COVID-19 pandemic: A systematic review and meta-analysis. Brain Behav Immun 2020; 88:901907.

22. Jones JH, Salathe M. Early assessment of anxiety and behavioral response to novel swine-origin influenza A (H1N1). PLoS one 2009; 4(12):e8032.

23. Schwartz J, King CC, Yen MY. Protecting healthcare workers during the coronavirus disease 2019 (COVID-19) outbreak: lessons from Taiwan's severe acute respiratory syndrome response. Clin Infect Dis 2020; 71(15):858-860.

24. Huang J, Liu F, Teng Z, Chen J, Zhao J, Wang X, Wu R. Care for the psychological status of frontline medical staff fighting against COVID-19. Clin Infect Dis 2020; ciaa385.

25. Moher D, Liberati A, Tetzlaff J, Altman DG, Prisma Group. Preferred reporting items for systematic reviews and meta-analyses: the PRISMA statement. PLoS Med 2009; 6(7):e1000097.

26. The modified Newcastle Ottawa scale for cross sectional studies [Internet]. [acessado 2020 Ago 1]. Disponível em: http://journals.plos.org/plosone/article/ file?id=info\%3Adoi/10.1371/journal.pone.0136065. s004\&type $=$ supplementary

27. Cochran WG. The combination of estimates from different experiments. Biometrics 1954; 10(1):101-129.

28. Higgins JP, Thompson SG, Deeks JJ, Altman DG. Measuring inconsistency in meta-analyses. BMJ 2003; 327(7414):557-560.

29. Campbell M, McKenzie JE, Sowden A, Katikireddi SV, Brennan SE, Ellis S, Hartmann-Boyce J, Ryan R, Shepperd S, Thomas J, Welch V. Synthesis without meta-analysis (SWiM) in systematic reviews: reporting guideline. BMJ 2020; 368:16890.

30. Apisarnthanarak A, Apisarnthanarak P, Siripraparat C, Saengaram P, Leeprechanon N, Weber DJ. Impact of Anxiety and Fear for COVID-19 Toward Infection Control Practices Among Thai Healthcare Workers. Infect Control Hosp Epidemiol 2020; 41(9):1093-1094.
31. Badahdah A, Khamis F, Al Mahyijari N, Al Balushi M, Al Hatmi H, Al Salmi I, Albulushi Z, Al Noomani J. The mental health of health care workers in Oman during the COVID-19 pandemic. Int J Soc Psychiatry 2020; 20764020939596.

32. Chatterjee SS, Bhattacharyya R, Bhattacharyya S, Gupta S, Das S, Banerjee BB. Attitude, practice, behavior, and mental health impact of COVID-19 on doctors. Indian J Psychiatry 2020; 62(3):257.

33. Chen Y, Zhou H, Zhou Y, Zhou F. Prevalence of self-reported depression and anxiety among pediatric medical staff members during the COVID-19 outbreak in Guiyang, China. Psychiatry Res 2020; 288:113005.

34. Chew QH, Chia FL, Ng WK, Lee WC, Tan PL, Wong CS, Puah SH, Shelat VG, Seah EJ, Huey CW, Phua EJ. Psychological and coping responses to COVID-19 amongst residents in training across ACGME-I accredited specialties in Singapore. Psychiatry Res 2020; 290:113146.

35. Civantos AM, Byrnes Y, Chang C, Prasad A, Chorath K, Poonia SK, Jenks CM, Bur AM, Thakkar P, Graboyes EM, Seth R. Mental health among otolaryngology resident and attending physicians during the COVID-19 pandemic: National study. Head Neck 2020; 42(7):1597-1609.

36. Consolo U, Bellini P, Bencivenni D, Iani C, Checchi V. Epidemiological Aspects and Psychological Reactions to COVID-19 of Dental Practitioners in the Northern Italy Districts of Modena and Reggio Emilia. Int J Environ Res Public Health 2020; 17(10):3459.

37. Corbett GA, Milne SJ, Mohan S, Reagu S, Farrell T, Lindow SW, Hehir MP, O'Connell MP. Anxiety and Depression Scores in Maternity Healthcare Workers during the Covid-19 Pandemic. Int J Gynaecol Obstet 2020; 151(2):297-298.

38. Dal'Bosco EB, Floriano LSM, Skupien SV, Arcaro G, Martins AR, Anselmo ACC. Mental health of nursing in coping with COVID-19 at a regional university hospital. Rev Bras Enferm 2020; 73(Supl. 2):e20200434.

39. Du J, Dong L, Wang T, Yuan C, Fu R, Zhang L, Liu B, Zhang M, Yin Y, Qin J, Bouey J. Psychological symptoms among frontline healthcare workers during COVID-19 outbreak in Wuhan. Gen Hosp Psychiatry 2020; 67:144-145.

40. Elbay RY, Kurtulmuş A, Arpacioğlu S, Karadere E. Depression, anxiety, stress levels of physicians and associated factors in Covid-19 pandemics. Psychiatry Res 2020; 290:113130.

41. Gupta AK, Mehra A, Niraula A, Kafle K, Deo SP, Singh B, Sahoo S, Grover S. Prevalence of anxiety and depression among the healthcare workers in Nepal during the COVID-19 pandemic. Asian J Psychiatr 2020; 54:102260.

42. 42. Hu D, Kong Y, Li W, Han Q, Zhang X, Zhu LX, Wan SW, Zuofeng LI, Qu SH, Jingqiu YA, Hong-Gu HE. Frontline nurses' burnout, anxiety, depression, and fear statuses and their associated factors during the COVID-19 outbreak in Wuhan, China: A largescale cross-sectional study. EClinicalMedicine 2020; 24:100424.

43. 43. Huang JZ, Han MF, Luo TD, Ren AK, Zhou XP. Mental health survey of medical staff in a tertiary infectious disease hospital for COVID-19. Zhonghua Lao Dong Wei Sheng Zhi Ye Bing Za Zhi 2020; 38(3):192-195. 
44. Lai J, Ma S, Wang Y, Cai Z, Hu J, Wei N, Wu J, Du H, Chen T, Li R, Tan H. Factors associated with mental health outcomes among health care workers exposed to coronavirus disease 2019. JAMA Netw Open 2020; 3(3):e203976.

45. Lin K, Yang BX, Luo D, Liu Q, Ma S, Huang R, Lu W, Majeed A, Lee Y, Lui LM, Mansur RB. The mental health effects of COVID-19 on health care providers in China. Am J Psychiatry 2020; 177(7):635-636.

46. Liu CY, Yang YZ, Zhang XM, Xu X, Dou QL, Zhang WW, Cheng AS. The prevalence and influencing factors in anxiety in medical workers fighting COVID-19 in China: a cross-sectional survey. Epidemiol Infect 2020; 148:e98.

47. Lu W, Wang H, Lin Y, Li L. Psychological status of medical workforce during the COVID-19 pandemic: A cross-sectional study. Psychiatry Res 2020; 288:112936.

48. Moghadasi AN. Evaluation of the Level of Anxiety among Iranian Multiple Sclerosis Fellowships During the Outbreak of COVID-19. Arch Iran Med 2020; 23(4):283.

49. Naser AY, Dahmash EZ, Al-Rousan R, Alwafi H, Alrawashdeh HM, Ghoul I, Abidine A, Bokhary MA, Al-Hadithi HT, Ali D, Abuthawabeh R, Abdelwahab GM, Alhartani YJ, Al Muhaisen H, Dagash A, Alyami HS. Mental health status of the general population, healthcare professionals, and university students during 2019 coronavirus disease outbreak in Jordan: A cross-sectional study. Brain Behav 2020; 10(8):e01730.

50. Que J, Le Shi JD, Liu J, Zhang L, Wu S, Gong Y, Huang W, Yuan K, Yan W, Sun Y, Ran M. Psychological impact of the COVID-19 pandemic on healthcare workers: a cross-sectional study in China. Gen Psychiatry 2020; 33(3):e100259.

51. Rossi R, Socci V, Pacitti F, Di Lorenzo G, Di Marco A, Siracusano A, Rossi A. Mental Health Outcomes Among Frontline and Second-Line Health Care Workers During the Coronavirus Disease 2019 (COVID-19) Pandemic in Italy. JAMA Net Open 2020; 3(5):e2010185.

52. Shechter A, Diaz F, Moise N, Anstey DE, Ye S, Agarwal S, Birk JL, Brodie D, Cannone DE, Chang B, Claassen J. Psychological distress, coping behaviors, and preferences for support among New York healthcare workers during the COVID-19 pandemic. Gen Hosp Psychiatry 2020; 66:1-8.

53. Tan BY, Chew NW, Lee GK, Jing M, Goh Y, Yeo LL, Zhang K, Chin HK, Ahmad A, Khan FA, Shanmugam GN, Chan BPL, Sunny S, Chandra B, Ong JJY, Paliwal PR, Wong LYH, Sagayanathan R, Chen JT, Ng AYY, Teoh HL, Ho CS, Ho RC, Sharma VK. Psychological impact of the COVID-19 pandemic on health care workers in Singapore. Ann Intern Med 2020; 173(4):317-320.

54. Temsah MH, Al-Sohime F, Alamro N, Al-Eyadhy A, Al-Hasan K, Jamal A, Al-Maglouth I, Aljamaan F, Al Amri M, Barry M, Al-Subaie S. The psychological impact of COVID-19 pandemic on health care workers in a MERS-CoV endemic country. J Infect Public Health 2020; 13(6):877-882.

55. Vanni G, Materazzo M, Santori F, Pellicciaro M, Costesta M, Orsaria P, Cattadori F, Pistolese CA, Perretta T, Chiocchi M, Meucci R. The Effect of Coronavirus (COVID-19) on Breast Cancer Teamwork: A Multicentric Survey. In Vivo 2020; 34(3 Supl.):1685-1694.
56. Wang S, Xie L, Xu Y, Yu S, Yao B, Xiang D. Sleep disturbances among medical workers during the outbreak of COVID-2019. Occup Med (Lond) 2020; 70(5):364369.

57. Xiao X, Zhu X, Fu S, Hu Y, Li X, Xiao J. Psychological impact of healthcare workers in China during COVID-19 pneumonia epidemic: A multi-center cross-sectional survey investigation. J Affect Disord 2020; 274:405-410.

58. Yang S, Kwak SG, Ko EJ, Chang MC. The mental health burden of the covid-19 pandemic on physical therapists. Int J Environ Res Public Health 2020; 17(10):3723.

59. Zandifar A, Badrfam R, Khonsari NM, Assareh M, Karim H, Azimzadeh M, Sepehr MN, Tajbakhsh R, Rahimi F, Ghanipour N, Agoushi A, Gelsefid SH, Etemadi F, Qorbani M. COVID-19 and medical staff's mental health in educational hospitals in Alborz Province, Iran. Psychiatry Clin Neurosci 2020; 74(9):499-501

60. Zhang C, Yang L, Liu S, Ma S, Wang Y, Cai Z, Du H, Li R, Kang L, Su M, Zhang J, Liu Z, Zhang B. Survey of insomnia and related social psychological factors among medical staff involved in the 2019 novel coronavirus disease outbreak. Front Psychiatry 2020; 11:306.

61. Zhang SX, Liu J, Jahanshahi AA, Nawaser K, Yousefi A, Li J, Sun S. At the height of the storm: Healthcare staff's health conditions and job satisfaction and their associated predictors during the epidemic peak of COVID-19. Brain Behav Immun 2020; 87:144-146.

62. Zhang WR, Wang K, Yin L, Zhao WF, Xue Q, Peng M, Min BQ, Tian Q, Leng HX, Du JL, Chang H. Mental health and psychosocial problems of medical health workers during the COVID-19 epidemic in China. Psychother Psychosom 2020; 89(4):242-250.

63. Zhao N, Huang Y. Chinese mental health burden during COVID-19 outbreak: a web-based cross-sectional survey. Asian J Psychiatr 2020; 51:102052.

64. Zhu Z, Xu S, Wang H, Liu Z, Wu J, Li G, Miao J, Zhang C, Yang Y, Sun W, Zhu S. COVID-19 in Wuhan: Sociodemographic characteristics and hospital support measures associated with the immediate psychological impact on healthcare workers. EClinicalMedicine 2020; 24:100443.

65. Zhu J, Sun L, Zhang L, Wang H, Fan A, Yang B, Li W, Xiao S. Prevalence and Influencing Factors of Anxiety and Depression Symptoms in the First-Line Medical Staff Fighting Against COVID-19 in Gansu. Front Psychiatry 2020; 11:386.

66. Spitzer RL, Kroenke K, Williams JB, Löwe B. A brief measure for assessing generalized anxiety disorder the GAD-7. Arch Intern Med 2006; 166(10):10921097.

67. Lovibond SH, Lovibond PF. Manual for the Depression Anxiety Stress Scales. Sydney: Psychology Foundation; 1995.

68. Zung WWK. A rating instrument for anxiety disorders. Psychosomatics 1971; 12:371-379.

69. Kroenke K, Spitzer RL, Williams JB, Monahan PO, Löwe B. Anxiety disorders in primary care: prevalence, impairment, comorbidity, and detection. Ann Intern Med 2007; 146:317-325.

70. Kroenke K, Spitzer RL, Williams JB, Löwe B. An ultra-brief screening scale for anxiety and depression: the PHQ-4. Psychosomatics 2009; 50:613-621. 
71. Beck AT, Epstein N, Brown G, Steer RA. An inventory for measuring clinical anxiety: psychometric properties. J Consult Clin Psychol 1988; 56(6):893.

72. Zigmond AS, Snaith RP. The hospital anxiety and depression scale. Acta Psychiatr Scand 1983; 67(6):361370.

73. Hamilton M. The assessment of anxiety states by rating. Br J Med Psychol 1959; 32:50-55.

74. Remes O, Brayne C, Van Der Linde R, Lafortune L. A systematic review of reviews on the prevalence of anxiety disorders in adult populations. Brain Behav 2016; 6(7):e00497.

75. Salari N, Hosseinian-Far A, Jalali R, Vaisi-Raygani A, Rasoulpoor S, Mohammadi M, Rasoulpoor S, Khaledi-Paveh B. Prevalence of stress, anxiety, depression among the general population during the COVID-19 pandemic: a systematic review and meta-analysis. Global Health 2020; 16(1):57.

76. Asher M, Asnaani A, Aderka IM. Gender differences in social anxiety disorder: A review. Clin Psychol Rev 2017; 56:1-12

77. Jalnapurkar I, Allen M, Pigott T. Sex differences in anxiety disorders: A review. HSOA J Psychiatry Depress Anxiety 2018; 4:012.

78. World Health Organization (WHO). Depression and other common mental disorders: global health estimates. Genebra: WHO; 2017.

79. Li SH, Graham BM. Why are women so vulnerable to anxiety, trauma-related and stress-related disorders? The potential role of sex hormones. Lancet Psychiatry 2017; 4(1):73-82.

80. Cross SE, Madson L. Models of the self: self-construals and gender. Psychol Bull 1997; 122(1):5.

81. Del Campo MT, Romo PE, de la Hoz RE, Villamor JM, Mahíllo-Fernández I. Anxiety and depression predict musculoskeletal disorders in health care workers. Arch Environ Occup Health 2017; 72(1):39-44.

82. Salehi H, Pennathur PR, Da Silva JP, Herwaldt LA. Examining health care personal protective equipment use through a human factors engineering and product design lens. Am J Infect Control 2019; 47(5):595-598.

83. Sang AX, Tisdale RL, Nielsen D, Loica-Mersa S, Miller T, Chong I, Shieh L. How Much Time are Physicians and Nurses Spending Together at the Patient Bedside? J Hosp Med 2019; 14(8):468-473.

84. Butler R, Monsalve M, Thomas GW, Herman T, Segre AM, Polgreen PM, Suneja M. Estimating time physicians and other health care workers spend with patients in an intensive care unit using a sensor network. Am J Med 2018; 131(8):972.e9-972.e15.

85. Wang J, Zhou M, Liu F. Reasons for healthcare workers becoming infected with novel coronavirus disease 2019 (COVID-19) in China. J Hosp infect 2020; 105(1):100-101.
86. Zhang M, Zhou M, Tang F, Wang Y, Nie H, Zhang L, You G. Knowledge, attitude, and practice regarding COVID-19 among healthcare workers in Henan, China. J Hosp infect 2020; 105(2):183-187.

87. Emanuel EJ, Persad G, Upshur R, Thome B, Parker M, Glickman A, Zhang C, Boyle C, Smith M, Phillips JP. Fair allocation of scarce medical resources in the time of Covid-19. N Engl J Med 2020; 382:2049-2055.

88. Adams JG, Walls RM. Supporting the health care workforce during the COVID-19 global epidemic. JAMA 2020; 323(15):1439-1440.

89. Dy LF, Rabajante JF. A COVID-19 Infection Risk Model for Frontline Health Care Workers. Netw Model Anal Health Inform Bioinform 2020; 9(1):57.

90. Liu X, Kakade M, Fuller CJ, Fan B, Fang Y, Kong J, Guan Z, Wu P. Depression after exposure to stressful events: lessons learned from the severe acute respiratory syndrome epidemic. Compr Psychiatry 2012; 53(1):15-23.

91. Brooks SK, Dunn R, Amlôt R, Rubin GJ, Greenberg N. A systematic, thematic review of social and occupational factors associated with psychological outcomes in healthcare employees during an infectious disease outbreak. J Occup Environ Med 2018; 60(3):248-257.

92. Kong X, Zheng K, Tang M, Kong F, Zhou J, Diao L, Wu S, Jiao P, Su T, Dong Y. Prevalence and factors associated with depression and anxiety of hospitalized patients with COVID-19. MedRxiv 2020; preprint.

Artigo apresentado em 11/08/2020

Aprovado em 06/11/2020

Versão final apresentada em 08/11/2020

Editores chefes: Romeu Gomes, Antônio Augusto Moura da Silva 